REVISTA MATEMATLCA de la

Universidad Complutense de Madrid

Volumen 6, número 1; 1993.

http://dx.doi.org/10.5209/rev_REMA.1993.v6.n1.17850

\title{
Remarks on Bases in a Fréchet Function Space
}

\author{
PÄIVI MATTILA* AND JARI TASKINEN
}

ABSTRACT. We present an explicit construction of a Schauder basis in the space $C(\mathbf{R}) \cap$ $L_{p}(\mathbf{R})$ for all $p, 1 \leq p<\infty$. This basis cannot be unconditional, since we give a proof of the fact that the Banach spaces $C(0,1)$ or $L_{1}(0,1)$ cannot be embedded in a Fréchet space with an unconditional basis.

\section{INTRODUCTION}

The purpose of this paper is to study questions concerning bases in the Fréchet function space $C(\mathbf{R}) \cap L_{p}(\mathbf{R})$ of continuous $L_{p}$-integrable functions from $\mathbf{R}$ into $\mathbb{K}(\mathbb{K}=\mathbf{R}$ or $\mathbf{C}$ ), $1 \leq p<\infty$. In Theorem 3 we show that $C(\mathbf{R}) \cap L_{p}(\mathbf{R})$ has a Schauder basis for all $p, 1 \leq p<\infty$. This basis cannot be unconditional, since we prove in theorem 4 that the Banach spaces $C(0,1)$ or $L_{1}(0,1)$ cannot be embedded in a Fréchet space with an unconditional basis. This result generalizes the corresponding, well-known statement for Banach spaces.

\footnotetext{
* This paper forms a part of the forthcoming licenciate's thesis of the first named author written at the Department of Mathematics, University of Helsinki.
}

1991 Mathematics Subject Classification: 46A06, 46E10

Editorial Complutense. Madrid, 1993. 
The study of these spaces is motivated by the following facts.

1. Taking an intersection is, of course, an elementary and concrete way to define a space, the elements of which have properties inherited from both of the original spaces. In general, spaces defined using an intersection now and then occur in analysis, although their linear topological structure usually does not play any role (see, e.g. $[\mathrm{H}]$, Theorems $2.2 .6,3.8 .2,7.3 .1)$. Our study of spaces $C(\mathbb{R}) \cap L_{p}(\mathbb{R})$ is meant as examples (rather than a systematic research) of phenomena occurring in the structure of these kind of spaces.

2. The space $C(\mathbb{R}) \cap L_{p}(\mathbb{R})$ is easily seen to be isomorphic to the subspace $E$ of $C(\mathbf{R}) \times L_{p}(\mathbf{R})$ spanned by elements of the form $(f f f)$. Clearly, because of its simple and concrete definition, $E$ is in a sense a natural subspace of $C(\mathbf{R}) \times L_{p}(\mathbf{R})$. Moreover, $C(\mathbf{R}) \times L_{p}(\mathbf{R})$ is isomorphic to a complemented subspace of $C\left(\mathbb{R}, L_{p}(0,1)\right)$, the space of continuous, $L_{p}$ valued functions on $\mathbb{R}$. So, the study of the spaces $C(\mathbf{R}) \cap L_{p}(\mathbb{R})$ is connected to the study of the above mentioned standard spaces of analysis.

3. The structure of the spaces $C(\mathbf{R}) \cap L_{p}(\mathbf{R})$ is quite complicated and thus interesting in itself, see [T] and [BT].

We now turn to the notations, definitions and preliminary results used in this paper. If $\Omega \subset \mathbb{R}^{n}, n \geq 1$, is an open set, we denote by $C(\Omega) \cap L_{p}(\Omega)$, $1 \leq p<\infty$, the Fréchet space of continuous, $L_{p}$-integrable functions from $\Omega$ into $\mathbb{R}$. The topology of this space is determined by the seminorms

$$
\begin{gathered}
p_{0}(f)=\left(\int_{\Omega}|f|^{p}\right)^{1 / p}, \\
p_{k}(f)=\sup _{x \in \Omega_{k}}|f(x)|, \quad k \in \mathbb{N}
\end{gathered}
$$

where $\left(\Omega_{k}\right)_{k=1}^{\infty}$ is an increasing sequence of compact subsets of $\Omega$, whose 
union is $\Omega$. For more details on these spaces we refer to $[\mathrm{BT}]$.

A sequence $\left(e_{n}\right)_{n=1}^{\infty}$ in a Fréchet space $E$ is called a (Schauder) basis, if every $x \in E$ has a unique representation as a convergent series

$$
x=\sum_{n=1}^{\infty} x_{n} e_{n}
$$

where $x_{n} \in \mathbb{K}$. The basis $\left(e_{n}\right)_{n=1}^{\infty}$ is called unconditional, if this series converges unconditionally. The sequence $\left(e_{n}\right)_{n=1}^{\infty} \subset E$ is a basic sequence, if it is a basis in its closed linear span. We refer to [J], Chapter 14 for more details.

We shall need a special example of basis called the Franklin system $\left(f_{n}\right)_{n=0}^{\infty}$. The elements $f_{n}$, the Franklin functions, are continuous real valued functions on the closed interval $I:=[0,1]$. The Franklin system is obtained applying the Gram-Schmidt orthogonalization procedure in $L_{2}(0,1)$ to the Schauder system, which is a basis in $C(0,1)$, see [LT], p. 4 , or especially [KS], VI.3 and VI.1. The Franklin system is a basis in $C(0,1)$ and $L_{p}(0,1)$ for $1 \leq p<\infty$, and it is an orthonormal basis in $L_{2}(0,1)$, see [KS], Theorems VI.6 and 7. Note that if

$$
g=\sum_{n=0}^{\infty} g_{n} f_{n}
$$

then the coefficients $\left(g_{n}\right)$ can be calculated in all of the above mentioned spaces from

$$
g_{n}=\int_{0}^{1} g f_{n}
$$

We shall need a result of Ciesielski [C], Theorem 1 , see also [KS], Corollary VI.4. Let $n \in \mathbb{N} \cup\{0\}$ and let $z_{n} \in[0,1]$ be defined by $z_{0}=0$, $z_{1}=1$, and for $n>1$,

$$
z_{n}=(2 i-1) / 2^{k+1}
$$


where $k$ and $i$ are such that $k \in\{0\} \cup \mathbb{N}, i \in \mathbb{N}, 1 \leq i \leq 2^{k}$ and $n=2^{k}+i$.

Theorem 1. Let the numbers $z_{n}, n \in N$, be as in (1.2). There exist absolute constants $C>0$ and $d, 0<d<1$ such that

$$
\left|f_{n}(t)\right| \leq C n^{1 / 2} d^{n\left|t-z_{n}\right|}
$$

for all $n \in \mathbb{N}$ and all $t \in\{0,1]$.

\section{EXISTENCE OF BASIS: AN EXPLICIT CONSTRUCTION}

In this section we show that $C(\mathbf{R}) \cap L_{p}(\mathbf{R})$ has a Schauder basis for all $p, 1 \leq p<\infty$. The strategy of the proof is as follows: we show that the Franklin system is a basis in the space $C_{\text {loc }}(0,1) \cap L_{p}(0,1)$, which is the space of continuous $L_{p}$-integrable functions on the open interval ]0,1[. It is then easy to see that this space is isomorphic to $C(\mathbf{R}) \cap L_{p}(\mathbf{R})$ so that we get the desired basis.

We define $C_{\mathrm{loc}}(0,1)$ as the Fréchet space of continuous $\mathbb{R}$-valued functions on the open interval $10,1[$, endowed with the seminorms

$$
q_{k}(f)=\sup _{x \in \Omega_{k}}\{f(x) \mid\}
$$

where $k \in \mathbb{N}$ and

$$
\Omega_{k}=[1 / k, 1-1 / k]
$$

The algebraic definition of $C_{\mathrm{l} \propto}(0,1) \cap L_{p}(0,1), 1 \leq p<\infty$, was already mentioned above. The topology of this Fréchet space is determined by the increasing system of norms

$$
\left(q_{k}+\|\cdot\|_{p}\right)_{k=1}^{\infty},
$$

where $\|f\|_{p}=\left(\int_{0}^{1}|f(x)|^{p} d x\right)^{1 / p}$. 
Theorem 2. The Franklin system $\left(f_{n}\right)_{n=0}^{\infty}$ is a Schauder basis in $C_{\text {lac }}(0,1) \cap L_{p}(0,1)$.

Strictly speaking, the elements of the Franklin system are functions defined on the closed interval $[0,1]$. Here we take the restriction of each $f_{n}$ to $] 0,1[$. This does not cause any confusions.

Proof. Let $g \in C_{\text {loc }}(0,1) \cap L_{p}(0,1)$; it is not a restriction to assume $\|g\|_{p}=1$. Since $\left(f_{n}\right)_{n=0}^{\infty}$ is an $L_{2}(0,1)$-orthonormal basis in $L_{p}(0,1), g$ has an $L_{p}(0,1)$-convergent representation

$$
g=\sum_{n=0}^{\infty} g_{n} f_{n}
$$

Recall that the coefficients are uniquely determined by

$$
g_{n}=\int_{0}^{1} g f_{n} .
$$

So the only thing is to prove that $(2.4)$ converges also in $C_{\mathrm{loc}}(0,1)$.

Let $k \in \mathbb{N}$ and $\varepsilon>0$. Our aim is to find $n_{0} \in \mathbb{N}$ such that

$$
q_{k}\left(g-\sum_{n=0}^{N} g_{n} f_{n}\right)<\varepsilon
$$

for all $N \geq n_{0}$, which proves the theorem. Let the constants $d, 0<d<1$, and $C>0$ be as in Theorem 1.1. There exists an $m \in \mathbb{N}$ such that the following conditions are satisfied:

$$
\begin{aligned}
& 1^{\mathrm{o}} m>2 k \\
& 2^{\mathrm{o}} d^{-m / 8 k}>m \\
& 3^{\mathrm{o}} C^{2} d^{n+/ 8 k}<\varepsilon / 64
\end{aligned}
$$

Moreover, since continuous functions form a dense subspace of $L_{p}$, we can find $\hat{g} \in C(0,1)$ such that 


$$
\hat{g}(x)=g(x) \text { for } x \in \Omega_{2 m}
$$

and

$$
\|\hat{g}-g\|_{p}<\varepsilon 4^{-1}\left(2 C^{\prime}(C+1)+C^{2} \sum_{n=1}^{\infty} n d^{n / 2 m}\right)^{-1},
$$

where $C^{\prime}=\max \left\{1,\left\|f_{0}\right\|_{q},\left\|f_{1}\right\|_{q}\right\}, 1 / p+1 / q=1$. (We first take a $\tilde{g} \in$ $C(0,1)$ such that $\|\bar{g}-g\|_{p}$ is smaller than, say, half of the number occurring on the right hand side of (2.8). Then we take an open interval $V, \Omega_{2 m} \subset$ $V \subset[0,1]$, so that

$$
\left(\int_{n \Omega_{2 n}}|g|^{p}\right)^{1 / p}
$$

is small enough. We define $\hat{g}(x)=g(x)$, if $x \in \Omega_{2 m}$, and $\hat{g}(x)=\tilde{g}(x)$, if $x \notin V$ and extend $\hat{g}$ continuously as line segments to the components of $W \Omega_{2 m}$ ) Since the Franklin system is a basis also in $C(0,1)$, we can find a number $n_{0} \in \mathbb{N}$ such that

$$
q_{k}\left(\hat{g}-\sum_{n=0}^{N} \hat{g}_{H} f_{n}\right)<\varepsilon / 2
$$

for all $N \leq n_{0}$ where $\hat{g}_{n}=\int_{0}^{1} \hat{g} f_{n}$.

Let the sequence $\left(z_{n}\right)_{n=0}^{\infty} \subset[0,1]$ of dyadic points be as in (1.2). We divide $\mathbb{N}$ into two subsets $\mathbb{N}_{1}$ and $\mathbb{N}_{2}$ :

$$
\begin{aligned}
& n \in \mathbb{N}_{1} \Leftrightarrow z_{n} \in \Omega_{m} \cup\{0,1\}, \\
& n \in \mathbb{N}_{2} \Leftrightarrow z_{n} \notin \Omega_{m} \cup\{0,1\} .
\end{aligned}
$$

To prove (2.6) using (2.9) we now make some estimates concerning the numbers $g_{n}$ and $\hat{g}_{n}$ :

In the following considerations $q$ is defined by $1 / p+1 / q=1$, In the case $q=\infty$ integrals must be replaced by suprema in the usual way. 
(a) For $n \in\{0,1\}$ we get (see (2.5) etc.) by the Hölder inequality

$$
\left|g_{n}-\hat{g}_{n}\right|=\left|\int_{0}^{1}(g-\hat{g}) f_{n}\right| \leq\|g-\hat{g}\|_{p}\left\|f_{n}\right\|_{q} \leq C^{\prime}\|g-\hat{g}\|_{p},
$$

where $C^{\prime}=\max \left\{1,\left\|f_{0}\right\|_{q},\left\|f_{1}\right\|_{q}\right\}$.

b) Assume that $n \in \mathbb{N}_{1} \backslash\{0,1\}$. Since $\hat{g}(x)=g(x)$ for $x \in \Omega_{2 m}$ (see (2.7)), we get

$$
\begin{aligned}
\left|g_{n}-\hat{g}_{n}\right| & =\left|\int_{0}^{1}(g-\hat{g}) f_{n}\right| \leq \int_{\Omega_{3 m}}|g-\hat{g}|\left|f_{n}\right| \\
& \leq\left(\int_{N \Omega_{I m}}|g-\hat{g}|^{p}\right)^{1 / p}\left(\int_{N \Omega_{I m}}\left|f_{n}\right|^{q}\right)^{1 / q}
\end{aligned}
$$

Applying Theorem 1.1 to the latter factor we see that (2.12) is not larger than

$$
C\|g-\hat{g}\|_{\rho}\left(\int_{\Omega_{2 m}}\left|\sqrt{n} d^{n\left|x-z_{n}\right|}\right|^{q} d x\right)^{1 / 4}
$$

Since $z_{n} \in \Omega_{m}$ by assumption, we have here $\left|x-z_{n}\right| \geq 1 / 2 m$ for all $x \in$ $\Lambda \Omega_{2 m}$, see (2.2). So, we get the estimate

$$
\left|g_{n}-\hat{g}_{n}\right| \leq C\|g-\hat{g}\|_{p} \sqrt{n} d^{n / 2 m} .
$$

c) Assume that $n \in \mathbb{N}_{2}$. We have again by (2.5) and the Hölder inequality

$$
\left|g_{n}\right| \leq\|g\|_{p}\left\|f_{n}\right\|_{q}
$$

Using the normalization $\|g\|_{p}=1$, Theorem 1.1 and the choice $0<d<1$ we get from (2.15) 


$$
\left|g_{n}\right| \leq C\left(\int_{0}^{1}\left|n^{1 / 2} d^{n\left|x-z_{n}\right|}\right|^{q}\right)^{1 / q} \leq C n^{1 / 2}
$$

On the other hand, if $x \in \Omega_{k}$, we get from Theorem 1.1 and $1^{*}$

$$
\left|f_{n}(x)\right| \leq C n^{1 / 2} d^{n|\cdot|-z_{*} \mid} \leq C n^{1 / 2} d^{n / 2 k},
$$

since $z_{n} \notin \Omega_{m}$ and $0<d<1$. Combining (2.16) and (2.17) we see that

$$
\left|g_{n} f_{n}(x)\right| \leq C^{2} n d^{n / 2 k}
$$

holds for $n \in \mathbb{N}_{2}$ and $x \in \Omega_{k}$.

Replacing $g$ by $\hat{g}$ in the above argument yields also

$$
\left|\hat{g}_{n} f_{n}(x)\right| \leq 2 C^{2} n d^{n / 2 k}
$$

for all $n \in \mathbb{N}_{2}$ and $x \in \Omega_{k}$; note that $\|\hat{g}\|_{p} \leq 2$, because of $\|g\|_{p}=1$ and (2.8).

We are now ready to prove (2.6). Let $n_{0}$ be as in (2.9). We have for $N \geq n_{0}$, by $(2.7), 1^{2},(2.9),(2.18)$ and $(2.19)$

$$
\begin{gathered}
q_{k}\left(g-\sum_{n=0}^{N} g_{n} f_{n}\right)=q_{k}\left(\hat{g}-\sum_{n=0}^{N} g_{n} f_{n}\right) \\
\leq q_{k}\left(\hat{g}-\sum_{n=0}^{N} \hat{g}_{n} f_{n}\right)+q_{k}\left(\sum_{\substack{n=0 \\
n=0}}^{N}\left(g_{n}-\hat{g}_{n}\right) f_{n}\right)
\end{gathered}
$$




$$
\begin{aligned}
& +q_{k}\left(\sum_{\substack{n=0 \\
N \in N_{2}}}^{N} g_{n} f_{n}\right)+q_{k}\left(\sum_{\substack{n=0 \\
n \in N_{2}}}^{N} \hat{g}_{n} f_{n}\right) \\
& \leq \varepsilon / 2+\sum_{\substack{n=0 \\
n \in N_{1}}}^{N}\left|g_{n}-\hat{g}_{n}\right| q_{k}\left(f_{n}\right)+3 \sum_{\substack{n=0 \\
n \in N_{2}}}^{N} C^{2} n d^{n / 2 k},
\end{aligned}
$$

We can further estimate, using (2.11) and $(2,14)$, the fact that $q_{k}\left(f_{n}\right) \leq$ $C n^{1 / 2}$ (see Theorem 1.1) and (2.8)

$$
\sum_{\substack{n=0 \\ n \in N_{1}}}^{N}\left|g_{n}-\hat{g}_{n}\right| q_{k}\left(f_{n}\right) \leq\|g-\hat{g}\|_{p}\left(2 C^{\prime}(C+1)+C^{2} \sum_{n=2}^{\infty} n d^{n / 2 m}\right) \leq \varepsilon / 4
$$

To estimate the remaining term of $(2.20)$ we remark that the smallest number in $\mathbb{N}_{2}$ is necessarily larger than or equal to $m / 2$. This follows from (2.10) and the definition of the numbers $z_{n},(1.2)$. Hence, by $1^{\circ}, 2^{\mathrm{o}}$ and $3^{\mathrm{o}}$ above

$$
\begin{aligned}
& \sum_{\substack{n=0 \\
n \in \mathbb{K}_{2}}}^{N} C^{2} n d^{n / 2 k} \leq \sum_{n \geq m / 2} C^{2} n d^{n / 2 k} \leq C^{2} \sum_{n \geq m / 2} d^{n / 4 k} \\
& \leq C^{2} d^{m / 8 k} /\left(1-d^{m / 8 k}\right) \leq 2 C^{2} d^{m / 8 k}<\varepsilon / 32 .
\end{aligned}
$$

Combining (2.20), (2.21) and (2.22) we get (2.26).

Let $\varphi:] 0,1[\rightarrow \mathbf{R}$ be a continuously differentiable homeomorphism such that $\varphi^{\prime}(x)>0$ for all $\left.x \in\right] 0,1[$. It is elementary to see that the linear operator

$$
\varphi: f \rightarrow\left(\varphi^{\prime}\right)^{1 / p} f \circ \varphi
$$

is an isomorphism from $C(\mathbf{R}) \cap L_{p}(\mathbf{R})$ onto $C_{\text {loc }}(0,1) \cap L_{p}(0,1)$. This and Theorem 2.1 yield. 
Theorem 3. Let $\left(f_{n}\right)_{n=0}^{\infty}$ be the Franklin system and let the operator $\phi$ be as in (2.23). The sequence $\left(\hat{\varphi}^{-1} f_{n}\right)_{n=0}^{\infty}$ is a Schauder basis in $C(\mathbb{R}) \cap$ $L_{p}(\mathbb{R})$ for all $p, 1 \leq p<\infty$.

Remark. It might be possible to prove the existence of basis in $C(\mathbb{R})$ $\cap L_{p}(\mathbf{R})$ in the context of the general structure theory of Fréchet spaces, but this would probably lead to a less explicit construction than the one presented above. Note that in our case it is even possible to describe analytically the coefficient functionals, by combining (2.5) and (2.23).

\section{NONEXISTENCE OF UNCONDITIONAL BASIS}

In view of the properties of the space $C(I)$, it is natural to expect that $C(\mathbb{R}) \cap L_{p}(\mathbf{R})$ cannot have an unconditional basis. We now turn to the proof of this fact.

Clearly, $C(\mathbb{R}) \cap L_{p}(\mathbb{R})$ contains for all $p, 1 \leq p<\infty$, subspaces isomorphic to $C(I)$. For example, the range of the map $f \rightarrow \tilde{f}$ from $C(I)$ into $C(\mathbb{R}) \cap L_{p}(\mathbb{R})$ is such a subspace, if $\tilde{f}$ is defined by extending the function $x \rightarrow f(x), x \in I, x \rightarrow 0, x \notin]-1,2[$, linearly to ]-1,0[ and ]1,2[. The following fact now implies that $C(\mathbb{R}) \cap L_{p}(\mathbf{R})$ does not have an unconditional basis for any $1 \leq p<\infty$.

Theorem 4. The Banach space $C(I)$ is not isomorphic to any subspace of a Fréchet space with an unconditional basis.

Remarks. After the first version of this paper was submitted, we learned that J.C. Díaz already proved in his unpublished thesis [D] our Theorem 4. However, since Theorem 4 is so intimately connected with the properties of the spaces $C(\Omega) \cap L_{p}(\Omega)$, we feel that it is worthwhile to present the proof in detail.

The proof of Theorem 4 consists of a generalization of the corresponding statement in Banach spaces (see for example [LT], 1.d.1). 
Having a look at the proof presented in [LT], 1.d.l one finds that [K], Theorem 4.3, should be useful to prove our Theorem. However, the choice of a suitable basic sequence in the Fréchet space has to be done so carefully that Kalton's general result is not quite enough for our purposes. In any case we shall use his ideas and his perturbation method, Proposition 4.1 in $[\mathrm{K}]$.

We remark that Bellenot constructed examples of Fréchet-Montel spaces which cannot be embedded in any Fréchet space with an unconditional basis, see [B1], Theorem 4.1. On the other hand he also proved in [B2] that any Fréchet-Schwartz space is isomorphic to subspace of a Fréchet space with an unconditional basis.

Proof. We recall that if $\left(e_{n}\right)_{n=1}^{\infty}$ is an unconditional basis in a Fréchet space $\left(E,\left(p_{k}\right)_{k=1}^{\infty}\right)$, where $\left(p_{k}\right)_{k=1}^{\infty}$ is an increasing system of seminorms defining to the topology of $E$, then for all $k \in \mathbb{N}$ there exists $\alpha(k) \in \mathbb{N}$, $\alpha(k) \geq k$ and $C_{k} \geq 1$ such that

$$
p_{k}\left(\sum_{n=1}^{N} \varepsilon_{n} x_{n} e_{n}\right) \leq C_{k} p_{\alpha \alpha k}\left(\sum_{n=1}^{M} x_{n} e_{n}\right)
$$

for all $N, M \in \mathbb{N}, M \geq N, \varepsilon_{n}=0$ or $\pm 1, x_{n} \in \mathbb{R}$. It is perhaps not so easy to find condition (3.1) in the standard references exactly in the above form, but in any case (3.1) is a consequence of the definition of unconditional basis and [J], Theorem 14.6.1(4): these and the BanachSteinhaus theorem show that $\left(T_{\mathrm{r}}\right)_{\mathbf{E} \in D}$, where

$$
D=\prod_{n=1}^{\infty}\{-1,0,1\}
$$

and

$$
T_{\varepsilon}: \sum_{n=1}^{\infty} x_{n} e_{n} \rightarrow \sum_{n=1}^{\infty} \varepsilon_{n} x_{n} e_{n}, \varepsilon=\left(\varepsilon_{n}\right)_{n=1}^{\infty},
$$

is an equicontinuous family of linear maps on $E$. 
Suppose now that there exists a Fréchet space $E$ with a unconditional basis $\left(e_{n}\right)_{n=1}^{\infty}$ having a subspace $F$ isomorphic to $L_{1}(I)$. We denote by $T: L_{1}(I) \rightarrow F$ the corresponding isomorphism. Let $\left(e^{\prime}{ }_{n}\right)_{n=1}^{\infty} \subset E^{\prime}$ be the sequence of the coefficient functionals of $\left(e_{n}\right)_{n=1}^{\infty}$. We denote by $P_{n}, n \in \mathbb{N}$, $P_{n} \in L(E)$ the projection

$$
P_{n} x=\sum_{i=1}^{n}\left\langle x, e_{i}^{\prime}\right\rangle e_{i}
$$

We may assume that the topology of $E$ is determined by an increasing system of seminorms $\left(p_{k}\right)_{k=1}^{\infty}$ satisfying

$$
p_{1}(T x) \geq\|x\| \text {, for } x \in L_{1}(I) \text { and } p_{1}\left(P_{n} x\right) \leq p_{1}(x) \text { for } n \in \mathbb{N}, x \in E \text {. }
$$

(We replace $p_{1}$ by

$$
\underset{n}{x \rightarrow \sup _{n}} p_{1}\left(P_{n} x\right)
$$

if necessary.) Let $\left(r_{m}\right)_{m=1}^{\infty} \subset L_{1}(I)$ be the sequence of Rademacher functions, $r_{m}(t)=\operatorname{sgn}\left(\sin \left(2^{m} \pi t\right)\right)$. It is known and easy to see that the following is true:

$1^{\mathrm{o}} r_{m} f \rightarrow 0$ weakly in $L_{1}(I)$ as $m \rightarrow \infty$, for all $f \in L_{1}(I)$.

$2^{2}\left\|f+r_{m} f\right\|_{L_{1}} \rightarrow\|f\|_{L_{1}}$ in $L_{1}(I)$ as $m \rightarrow \infty$, for all $f \in L_{1}(I)$.

Multiplication is defined pointwise here. We choose the sequences $\left(x_{n}\right)_{n=1}^{\infty}$ $\subset L_{1}(I),\left(k_{k}\right)_{n=1}^{\infty} \subset \mathbb{N}$ and $\left(m_{n}\right)_{n=1}^{\infty} \subset \mathbb{N}$ inductively as follows. We denote $x_{0}=1, m_{0}=1$ and $k_{0}=0$. Assume that $n \in \mathbb{N}$ and that $x_{t}, m_{t}$ and $k_{t}$ are chosen for $t<n$ such that

$$
1 / 2<\left\|x_{1}+\ldots+x_{f}\right\|<2
$$

for all $t<n$. Since $r_{m} f \rightarrow 0$ weakly in $L_{1}(I)$ for all $f \in L_{1}(I)$, it is clear that $T\left(r_{m} f\right) \rightarrow 0$ weakly in $E$ for all $f$. Hence, we can find $m_{n}>m_{n=1}$ such that $x_{n}:=r_{m_{n}}\left(x_{1}+x_{2}+\ldots+x_{n-1}\right)$ satisfies 


$$
1 / 2<\left\|x_{1}+x_{2}+\ldots+x_{n}\right\|_{L_{1}}<2
$$

and

$$
\sum_{r=1}^{k_{n-1}}\left|\left\langle T x_{n}, e_{r}^{\prime}\right\rangle\right| p_{n}\left(e_{r}\right) \leq 2^{-n-4}
$$

We choose $k_{n}>k_{n-1}$ such that

$$
p_{n}\left(\sum_{t=k_{n}+1}^{\infty}\left|\left\langle T x_{n}, e_{t}^{\prime}\right\rangle\right| e_{t}\right) \leq 2^{-n-4}
$$

Since $\left|r_{m}(t)\right|=1$ for all $m$ and $t \in I$, we have $\left\|x_{n}\right\|_{L_{1}}=\| x_{1}+\ldots+$ $x_{n-1} \|_{L}$, so that (3.3) implies

$$
1 / 2<\left\|x_{n}\right\|_{L_{1}}<2
$$

for all $n$.

We define for all $n \in \mathbb{N}$

$$
z_{n}=\sum_{r=k_{n-1}+1}^{k_{n}}\left\langle T x_{n}, e_{f}^{\prime}\right\rangle e_{t},
$$

so that $\left(z_{n}\right)_{n=1}^{\infty}$ is a block basis of $\left(e_{n}\right)_{n=1}^{\infty}$ and thus a basic sequence in $E$. We denote by $\left(w_{n}\right)_{n=1}^{\infty} \subset E_{0}^{\prime}$ the sequence of coefficient functionals of $\left(z_{n}\right)_{n=1}^{\infty}$, where $E_{0}=\operatorname{sp}\left(z_{n} \mid n \in \mathbb{N}\right)$.

We claim that the following is true:

i) The series $\sum_{n=1}^{\infty}\left(z_{n}-T x_{n}\right)$ converges absolutely in $E$.

ii) The seminorm $p_{1}$ satisfies 


$$
\sum_{n=1}^{\infty} 2 p_{1}\left(z_{n}-T x_{n}\right)<1
$$

and

$$
\left|w_{n}(x)\right| \leq 8 p_{1}(x)
$$

for all $n \in \mathbb{N}$ and for all $x \in E_{0}$.

To prove i) let $k \in \mathbb{N}$. We have, by the choice of $z_{n}$ and (3.4) and (3.5)

$$
\begin{aligned}
& \sum_{n=1}^{\infty} p_{k}\left(z_{n}-T x_{n}\right) \\
& \leq \sum_{n<k} p_{k}\left(z_{n}-T x_{n}\right)+\sum_{n \geq k} p_{n}\left(\sum_{i=1}^{k_{n-1}}\left\langle T x_{n}, e_{t}^{\prime}\right\rangle e_{t}\right)+\sum_{n \geq k} p_{n}\left(\sum_{i=k_{n}+1}\left\langle T x_{n}, e_{i}^{\prime}\right\rangle_{i}\right) \\
& \leq \sum_{n<k} p_{k}\left(z_{n}-T x_{n}\right)+2 \sum_{n \geq k} 2^{-n-4} .
\end{aligned}
$$

The statement (3.8) follows directly from (3.4) and (3.5):

$$
p_{1}\left(z_{n}-T x_{n}\right) \leq p_{1}\left(\sum_{t=1}^{k_{n-1}}\left\langle T x_{n}, e_{t}^{\prime}\right\rangle e_{t}\right)+p_{1}\left(\sum_{i=k_{n}+1}^{\infty}\left\langle T x_{n}, e_{l}^{\prime}\right\rangle e_{t}\right) \leq 2^{-n-4}
$$

If $x=\Sigma_{n=1}^{\infty} \alpha_{n} z_{n} \in E_{0}$, we have, for all $n,\left|w_{n}(x)\right|=\left|\alpha_{n}\right|$, and on the other hand, by (3.2), (3.8) and (3.6), 


$$
\begin{aligned}
2 p_{1}(x) & \geq p_{1}\left(P_{k_{n}} x\right)+p_{1}\left(P_{k_{n+1}+1} x\right) \\
& \geq p_{1}\left(P_{k_{n}} x-P_{k_{n-1}+1} x\right)=\left|\alpha_{n}\right| p_{1}\left(z_{n}\right) \\
& \geq 2^{-1}\left|\alpha_{n}\right| p_{1}\left(T x_{n}\right) \geq 2^{-1}\left|\alpha_{n}\right|\left\|x_{n}\right\|_{L_{1}} \geq 4^{-1}\left|\alpha_{n}\right|
\end{aligned}
$$

This proves (3.9).

The facts i) and ii) above and the choice of $p_{1}$, (3.2), imply that $\left(T x_{n}\right)_{n=1}^{\infty}$ is a deformation of $\left(z_{n}\right)_{n=1}^{\infty}$ in the sense of [K], Proposition 4.1 (take $p_{1} / 8$ for $p_{0}$ in $[\mathrm{K}]$ ). So, $\left(T x_{n}\right)_{n=1}^{\infty}$ is a basic sequence equivalent to $\left(z_{n}\right)_{n=1}^{\infty}$, according to the result just cited. Hence, also $\left(x_{n}\right)_{n=1}^{\infty}$ and $\left(z_{n}\right)_{n=1}^{\infty}$ are equivalent unconditional basic sequences. By [A], Theorem 3, and (3.1), there exist indices $n_{0}, m$ and $n_{1}$, and positive constants $M_{1}, M_{m}$ and $C_{m}$ such that for all $N>n_{0}, n_{1}$

$$
\begin{aligned}
& \sup _{z_{n}= \pm 1} \sum_{n=n_{0}}^{N} \varepsilon_{n} x_{n} \|_{L_{1}} \leq M_{1} \sup _{z_{n}= \pm 1} \sup _{n_{0} \leq \leq \leq N}\left(\sum_{n=n_{0}}^{1} \varepsilon_{n} z_{n}\right) \\
& \leq M_{1} C_{m} p_{\alpha(m)}\left(\sum_{n=1}^{N} z_{n}\right) \\
& \leq M_{1} C_{m} p_{\alpha(m)}\left(\sum_{n=1}^{n_{1}} z_{n}\right)+C_{m} M_{1} M_{m} \sup \left\|\sum_{n_{1} \leq \leq \leq N}^{1} x_{n=n_{1}}\right\|_{L_{i}}
\end{aligned}
$$

Clearly, the first term here is independent of $N$. But also the second term has an upper bound independent of $N$, because of (3.3). On the other hand, by [W], Remark II.D.7, 


$$
\left(\sum_{n=1}^{N}\left\|x_{n}\right\|_{L_{1}}^{2}\right)^{1 / 2} \leq D \sup _{\varepsilon_{n} \neq \pm 1}\left\|\sum_{n=n_{0}}^{N} \varepsilon_{n} x_{n}\right\|_{L_{1}}
$$

for a constant $D>0$. Now (3.6) shows that this is a contradiction, since the left hand side of (3.11) does not have an upper bound independent of $N$.

So, we have shown that $L_{1}(I)$ is not isomorphic to any subspace of a Fréchet space with an unconditional basis. This implies our theorem, since $C(I)$ contains a subspace isomorphic to $L_{1}(I)$.

\section{References}

[A] ARSOVE, M. Similar Bases and isomorphisms in Fréchet spaces. Math. Ann. 135 (1958), 283-293.

[B1] BELLENOT, S. Basic sequences in non-Schwartz-Fréchet spaces. Trans. Amer. Math. Soc. 258.1 (1980), 199-216.

[B2] BELLENOT, S. Each Schwartz Fréchet space is a subspace of a Schwartz Fréchet space with an unconditional basis. Compos. Math. 42.3 (1981), 273-278.

[BT] BONET, J. and TASKINEN, J. Non-distinguished Fréchet function spaces. Bull. Soc. Roy. Sci. Liége 58.6 (1989), 483-490.

[C] CIESIELSKI, Z. Properties of the orthogonal Franklin system II. Studia Math. 27 (1966), 289-323.

[D] DIAZ, J.C. Subespacios y bases $K$-monotonas en los espacios escalonados de Köthe. Tesis Doctora], Granada (1985).

[H] HÖRMANDER, L. Linear partial differential operators. Springer, Berlin (1963).

[J] JARCHOW, H. Locally convex spaces. Teubner, Stuttgart (1981).

[K] KALTON, N. Normalization properties of Schauder bases. Proc. London Math. Soc. (3) 22 (1971), 91-105.

[KS] KASHIN, B. and SAAKYAN, A. Orthogonal series. Translations of mathematical monographs vol. 75, AMS, Providence (1989).

[LT] LINDENSTRAUSS, J. and TZAFIRI, L. Classical Banach spaces $I$. Springer, Berlin-Heidelberg-New York (1977). 
[T] TASKINEN, J. Examples of non-distinguished Fréchet spaces. Ann. Acad. Sci. Fenn. 14 (1989), 75-88.

[W] WOJTASZCZYK, P. Banach spaces for analysts. Cambridge University Press (1991).

University of Helsinki

Department of Mathematics

Hallituskatu 15

SF-00100 Helsinki

FINLAND
Recibido: 28 de agosto de 1992

Revisado: 10 de diciembre de 1992 